\title{
Multiple normalized solutions of Chern-Simons-Schrödinger system
}

\author{
Jianjun Yuan
}

Abstract. In this paper, we consider the following equation

$-\Delta u+\omega u+\left(\int_{|x|}^{\infty} \frac{h(s)}{s} u^{2}(s) d s\right) u+\frac{h^{2}(|x|)}{|x|^{2}} u-\lambda|u|^{p-2} u=0 \quad$ in $\mathbb{R}^{2}$,

for $p>2$ and $\lambda>0$, which appeared in Byeon et al. (J Funct Anal 263(6):1575-1608, 2012) to find the standing wave solutions of the ChernSimons-Schrödinger system. By using the minimax theorem, we get the multiplicity results for the $L^{2}$-normalized solutions to the equation, and thus there are multiple $L^{2}$-normalized solutions of the Chern-SimonsSchrödinger system.

Mathematics Subject Classification. 35Q55, 35A15, 35B30.

Keywords. Palais-Smale sequence, Euler-Lagrange equation, Pohozaev identity, Standing wave.

\section{Introduction}

We investigate the following Chern-Simons gauged nonlinear Schrödinger system

$$
\begin{aligned}
i D_{0} \phi+\left(D_{1} D_{1}+D_{2} D_{2}\right) \phi & =-\lambda|\phi|^{p-2} \phi, \\
\partial_{0} A_{1}-\partial_{1} A_{0} & =-\operatorname{Im}\left(\bar{\phi} D_{2} \phi\right), \\
\partial_{0} A_{2}-\partial_{2} A_{0} & =\operatorname{Im}\left(\bar{\phi} D_{1} \phi\right), \\
\partial_{1} A_{2}-\partial_{2} A_{1} & =-\frac{1}{2}|\phi|^{2},
\end{aligned}
$$

where $\phi$ is a complex scalar field and $A_{\mu}: \mathbb{R}^{2+1} \rightarrow \mathbb{R}$ is the gauge field, $D_{\mu}=\partial_{\mu}+i A_{\mu}$ is the covariant derivative for $\mu=0,1,2, \lambda>0$ and $p>2$ is a constant. 
The system (1.1)-(1.4) conserves the charge and the energy

$$
\begin{aligned}
M(t) & :=\|\phi(t, \cdot)\|_{L^{2}\left(\mathbb{R}^{2}\right)}^{2}=M(0), \\
E(t) & :=\frac{1}{2} \sum_{j=1}^{2} \int_{\mathbb{R}^{2}}\left|D_{j} \phi(t, \cdot)\right|^{2} d x-\lambda \frac{1}{p} \int_{\mathbb{R}^{2}}|\phi(t, \cdot)|^{p} d x=E(0) .
\end{aligned}
$$

The system (1.1)-(1.4) is invariant under the following gauge transformation

$$
\phi \rightarrow \phi e^{i \chi}, A_{\mu} \rightarrow A_{\mu}-\partial_{\mu} \chi
$$

where $\chi: \mathbb{R}^{1+2} \rightarrow \mathbb{R}$ is an arbitrary $C^{\infty}$ function.

This model was proposed to study vortex solutions, which carry both electric and magnetic charges. It has applications in high-temperature superconductivity, we refer the reader to $[9,15,16]$ for the physical background. For the mathematical study of this model, see e.g. [4,5,10-12,21].

In $[5,11,21]$, the authors consider the standing wave solution to (1.1)(1.4) for $p>2$ of the form

$$
\begin{aligned}
\phi(t, x) & =u(|x|) e^{i \omega t}, A_{0}(t, x)=k(|x|) \\
A_{1}(t, x) & =\frac{x_{2}}{|x|^{2}} h(|x|), A_{2}(t, x)=-\frac{x_{1}}{|x|^{2}} h(|x|),
\end{aligned}
$$

where $\omega>0$ is a given frequency and $u, k, h$ are real valued functions on $[0, \infty)$ with $h(0)=0$. Note the form of $A_{1}$ and $A_{2}$ satisfies the Couloumb gauge condition $\partial_{1} A_{1}+\partial_{2} A_{2}=0$. Substituting the ansatz (1.8) into the system (1.1)-(1.4), we get the following elliptic equation

$$
-\Delta u+\omega u+\left(\xi+\int_{|x|}^{\infty} \frac{h(s)}{s} u^{2}(s) d s\right) u+\frac{h^{2}(|x|)}{|x|^{2}} u-\lambda|u|^{p-2} u=0 \quad \text { in } \quad \mathbb{R}^{2},
$$

where $h(s)=\int_{0}^{s} \frac{l}{2} u^{2}(l) d l$ and $\xi=\lim _{|x| \rightarrow \infty} k(|x|)$.

Equation (1.9) is the the Euler-Lagrange equation of the functional

$$
\begin{aligned}
J(u):= & \frac{1}{2} \int_{\mathbb{R}^{2}}|\nabla u|^{2}+(\omega+\xi) u^{2}+\frac{u^{2}}{|x|^{2}}\left(\int_{0}^{|x|} \frac{s}{2} u^{2}(s) d s\right)^{2} d x \\
& -\frac{\lambda}{p} \int_{\mathbb{R}^{2}}|u|^{p} d x, u \in H_{r}^{1}\left(\mathbb{R}^{2}\right),
\end{aligned}
$$

Here $H_{r}^{1}\left(\mathbb{R}^{2}\right)$ denotes the set of radially symmetric functions in $H^{1}\left(\mathbb{R}^{2}\right)$.

In view of the transformation (1.7), by choosing $\chi=c t+n \pi$ for $c$ real and $n$ integer, we see, when

$$
\left(\phi, A_{0}, A_{1}, A_{2}\right)=\left(u(|x|) e^{i \omega t}, k(|x|), \frac{x_{2}}{|x|^{2}} h(|x|),-\frac{x_{1}}{|x|^{2}} h(|x|)\right)
$$

is a solution of (1.1)-(1.4), then so is

$$
\left(\tilde{\phi}, \tilde{A}_{0}, A_{1}, A_{2}\right)=\left((-1)^{|n|} u(|x|) e^{i(\omega+c) t}, k(|x|)-c, \frac{x_{2}}{|x|^{2}} h(|x|),-\frac{x_{1}}{|x|^{2}} h(|x|)\right) .
$$

We see that if we obtain a standing wave with a certain frequency, then we can get a standing wave with any frequency, and we say that $\left(\phi, A_{0}, A_{1}, A_{2}\right)$ 
and $\left(\tilde{\phi}, \tilde{A}_{0}, A_{1}, A_{2}\right)$ are gauge equivalent as the standing wave of the form (1.8). Correspondingly, if the two standing wave $\left(u e^{i \omega t}, A_{0}, A_{1}, A_{2}\right)$ and $\left(\tilde{u} e^{i \tilde{\omega} t}, \tilde{A}_{0}, \tilde{A}_{1}, \tilde{A}_{2}\right)$ are gauge equivalent, and

$$
\begin{aligned}
& -\Delta u+\omega u+\left(\xi+\int_{|x|}^{\infty} \frac{h(s)}{s} u^{2}(s) d s\right) u+\frac{h^{2}(|x|)}{|x|^{2}} u-\lambda|u|^{p-2} u=0, \\
& -\Delta \tilde{u}+\tilde{\omega} \tilde{u}+\left(\tilde{\xi}+\int_{|x|}^{\infty} \frac{\tilde{h}(s)}{s} \tilde{u}^{2}(s) d s\right) \tilde{u}+\frac{\tilde{h}^{2}(|x|)}{|x|^{2}} \tilde{u}-\lambda|\tilde{u}|^{p-2} \tilde{u}=0, \\
& h(s)=\int_{0}^{s} \frac{t}{2} u^{2}(t) d t, \tilde{h}(s)=\int_{0}^{s} \frac{t}{2} \tilde{u}^{2}(t) d t,
\end{aligned}
$$

for certain $\xi$ and $\tilde{\xi}$, then we have $\tilde{\xi}+\tilde{\omega}=\xi+\omega$. So to seek the non gauge equivalent standing wave, we will choose the boundary values $\xi$ and $\tilde{\xi}$ with $\omega+\xi \neq \tilde{\omega}+\tilde{\xi}$. In this paper, we just choose $\xi=\tilde{\xi}=0$, and correspondingly $\omega \neq \tilde{\omega}$.

We call a solution $u$ of the system (1.1)-(1.4) is normalized if for a fixed constant $c>0,\|u\|_{L^{2}\left(\mathbb{R}^{2}\right)}=c$. In view of the mass conservation law (1.5), it is natural to seek solutions of these type.

Recently, there are many papers on the Schrödinger-Poisson-Slater system

$$
-\Delta u+\lambda u+\left(|x|^{-1} *|u|^{2}\right) u-|u|^{p-2} u=0, \quad \text { in } \quad \mathbb{R}^{3},
$$

when $2<p \leq 6$, see e.g. $[1,2,6-8,13,17,20,22,26]$. In $[7,8,13]$, for $p$ in some ranges and $c>0$, by using the concentration-compactness method of Lions [18, 19], the authors obtained the minimizers on

$$
\tilde{S}(c):=\left\{u \in H^{1}\left(\mathbb{R}^{3}\right):\|u\|_{L^{2}\left(\mathbb{R}^{3}\right)}=c\right\}
$$

for the functional

$$
I(u):=\frac{1}{2} \int_{\mathbb{R}^{3}}|\nabla u|^{2} d x+\frac{1}{4} \iint_{\mathbb{R}^{3}} \frac{|u(x)|^{2}|u(y)|^{2}}{|x-y|} d x d y-\frac{1}{p} \int_{\mathbb{R}^{3}}|u|^{p} d x,
$$

and thus obtained normalized solutions of (1.11). When $\frac{10}{3}<p<6$, in [20], by using the methods developed for nonlinear Schrödinger equation in $[3,14]$, the author obtained there are infinitely many normalized solutions to the Schrödinger-Poisson-Slater system. Since our equation is similar to the Schrödinger-Poisson-Slater system in many parts, so we can use some ideas developed for it.

Now we focus on to seek the normalized solutions to (1.1)-(1.4).

The Eq. (1.9) now becomes

$$
-\Delta u+\omega u+\left(\int_{|x|}^{\infty} \frac{h(s)}{s} u^{2}(s) d s\right) u+\frac{h^{2}(|x|)}{|x|^{2}} u-\lambda|u|^{p-2} u=0 \quad \text { in } \quad \mathbb{R}^{2},
$$

and the solution $u$ is obtained as a critical point of the functional

$$
J(u):=\frac{1}{2}\|\nabla u\|_{L^{2}}^{2}+\frac{1}{2} \int_{\mathbb{R}^{2}} \frac{u^{2}}{|x|^{2}}\left(\int_{0}^{|x|} \frac{s}{2} u^{2}(s)\right)^{2} d x-\frac{\lambda}{p} \int_{\mathbb{R}^{2}}|u|^{p} d x,
$$


on the constraint

$$
\text { for } c>0, S(c):=\left\{u \in H_{r}^{1}\left(\mathbb{R}^{2}\right):\|u\|_{L^{2}\left(\mathbb{R}^{2}\right)}=c\right\} .
$$

In [5], by considering the minimization of $J(u)$ on $S(c)$, the authors obtained the following results: when $2<p \leq 3$, there exits a positive minimizer of $J(u)$ on $S(c)$; when $3<p<4$ and $c$ is small, there also exists a positive minimizer of $J(u)$ on $S(c)$. Here, by using the minimax theorems, we obtain the following multiplicity results when $p>2$ and $p \neq 4$.

Theorem 1.1. (1) If $2<p \leq 3$, then the Eq. (1.11) has infinitely many distinct pairs of radial solutions $\left(\omega_{n}, \pm u_{n}\right)$, satisfying $\left\|u_{n}\right\|_{L^{2}}=c, \omega_{n}>0$, for each $n \in \mathbb{N}$ and $c>0$.

(2) If $3<p<4$, then there exists $c_{0}>0$, such that for any $c \in\left(0, c_{0}\right)$, the Eq. (1.11) has infinitely many distinct pairs of radial solutions $\left(\omega_{n}, \pm u_{n}\right)$, $n \in \mathbb{N}$, satisfying $\left\|u_{n}\right\|_{L^{2}}=c, \omega_{n}>0$.

(3) If $p>4$, then there exists $c_{1}>0$, such that for any $c \in\left(0, c_{1}\right)$, the Eq. (1.11) has infinitely many distinct pairs of radial solutions $\left(\omega_{n}, \pm u_{n}\right)$, $n \in \mathbb{N}$, satisfying $\left\|u_{n}\right\|_{L^{2}}=c, \omega_{n}>0$, and $\left\|u_{n}\right\|_{H^{1}} \rightarrow \infty$, as $n \rightarrow \infty$.

In the Sect. 2, we will make some preparations which will be used in Sect. 3. In Sect. 3, in the $2<p<4$ case, we will use the Krasnoselski genus to constructs critical points; In the $p>4$ case, as for the nonlinear Schrödinger equation in [3] and Schrödinger-Poisson-Slater system in [20], we will use linking type theorem in [3] and the auxiliary function approach in [14] to construct bounded Palais-Smale sequences of (1.12). The linking type theorem in [3], is a variant of the fountain theorem, for more on the linking type theorem, we refer the reader to $[3,25]$. As in $[3,14,20]$, we define the auxiliary $J(H(u, m)): S(c) \times \mathbb{R} \rightarrow \mathbb{R}$, where $H(u, m)(x)=e^{m} u\left(e^{m} x\right)$ (see Sect. 3.1.2). The virtue of $J(H(u, m))$ compared to $J(u): S(c) \rightarrow \mathbb{R}$, is that we have an extra dimension $\mathbb{R}$, and because of this, for the corresponding P-S sequences, they have an extra restriction $\partial_{m} J(H(u, m)) \rightarrow 0$, which is related to Pohozaev identity. By combining this extra information, we can show that the constructed $\mathrm{P}-\mathrm{S}$ sequences are bounded. Then we consider the convergence of the Palais-Smale squences, and prove Theorem 1.1.

Notation: Throughout the article we will use the letter $C$ to denote various constants whose exact value may change from line to line. Recall that a sequence $\left(u_{m}\right)$ in a Banach space $V$ is a Palais-Smale sequence (abbreviated as $\mathrm{P}-\mathrm{S}$ sequence) for $E$ if $\mid E\left(u_{m} \mid \leq c\right.$, uniformly in $m$, while $\left\|D E\left(u_{m}\right)\right\| \rightarrow 0$ as $m \rightarrow \infty$, where $D E\left(u_{m}\right)$ is the Fréchet derivative of $E$ at $u_{m}$.

\section{Preliminaries}

Let us recall the Pohožaev identity Proposition 2.3 and compactness lemma Lemma 3.2 in [5].

Lemma 2.1. (Pohožaev identity) Let $b, c$ and $d$ be real constants and $u \in H_{r}^{1}$ be a weak solution of the equation: 


$$
\Delta u+b u+c\left(\int_{|x|}^{\infty} \frac{h(s)}{s} u^{2}(s) d s\right) u+c \frac{h^{2}(|x|)}{|x|^{2}} u+d|u|^{p-2} u=0, \quad \text { in } \quad \mathbb{R}^{2}
$$

where $h(s)=\int_{0}^{s} \frac{t}{2} u^{2}(t) d t$, then we have

$$
b \int_{\mathbb{R}^{2}} u^{2} d x+2 c \int_{\mathbb{R}^{2}} \frac{h^{2}(|x|)}{|x|^{2}} u^{2} d x+\frac{2 d}{p} \int_{\mathbb{R}^{2}}|u|^{p} d x=0 .
$$

Let $c(u)=\int_{\mathbb{R}^{2}} \frac{h^{2}(|x|)}{|x|^{2}} u^{2} d x$, with $h(s)=\int_{0}^{s} \frac{t}{2} u^{2}(t) d t$, then we have

Lemma 2.2. Suppose that a sequence $\left\{u_{n}\right\}$ converge weakly to a function $u$ in $H_{r}^{1}$ as $n \rightarrow \infty$. Then for each $\varphi \in H_{r}^{1}, c\left(u_{n}\right), c^{\prime}\left(u_{n}\right) \varphi$ and $c^{\prime}\left(u_{n}\right) u_{n}$ converges up to a subsequence to $c(u), c^{\prime}(u) \varphi$, and $c^{\prime}(u) u$, respectively, as $n \rightarrow \infty$.

\section{Lemma 2.3.}

$$
\int_{\mathbb{R}^{2}} \frac{h^{2}(|x|)}{|x|^{2}} u^{2} d x \leq C\|u\|_{L^{2}}^{4}\|\nabla u\|_{L^{2}}^{2} .
$$

Proof. By using Hölder's inequality and Gagliardo-Nirenberg inequality, we have

$$
\left(\frac{1}{|x|} \int_{B_{|x|}} u^{2} d y\right)^{2} \leq \int_{B_{|x|}} u^{4} d y \leq \int_{\mathbb{R}^{2}} u^{4} d x \leq C\|u\|_{L^{2}}^{2}\|\nabla u\|_{L^{2}}^{2}
$$

and

$$
\begin{aligned}
\int_{\mathbb{R}^{2}} \frac{h^{2}(|x|)}{|x|^{2}} u^{2} d x & \leq C \int_{\mathbb{R}^{2}} u^{2}\left(\frac{1}{|x|} \int_{B_{|x|}} u^{2} d y\right)^{2} d x \\
& \leq C\|u\|_{L^{2}}^{4}\|\nabla u\|_{L^{2}}^{2}
\end{aligned}
$$

Lemma 2.4. If $u \in H_{r}^{1}\left(\mathbb{R}^{2}\right)$ is a weak solution of

$$
-\Delta u+\left(\int_{|x|}^{\infty} \frac{h(s)}{s} u^{2}(s) d s\right) u+\frac{h^{2}(|x|)}{|x|^{2}} u-\lambda|u|^{p-2} u=\mu u \quad \text { in } \quad \mathbb{R}^{2},
$$

with $\mu \geq 0$.

(1) If $2<p \leq 3$, then $u \equiv 0$.

(2) If $3<p<4$, then there exists a constant $c_{0}>0$, such that, when $\|u\|_{2}^{2} \leq c_{0}$ and $J(u)<0, u \equiv 0$.

(3) If $p>4$, then there exists a constant $c_{1}>0$, such that, when $\|u\|_{2}^{2} \leq c_{1}$, $u \equiv 0$.

Proof. Multiply (2.3) by $u$ and integrate by parts, we have

$$
\int_{\mathbb{R}^{2}}|\nabla u|^{2} d x-\mu \int_{\mathbb{R}^{2}}|u|^{2} d x+3 \int_{\mathbb{R}^{2}} \frac{h^{2}(|x|)}{|x|^{2}} u^{2} d x-\lambda \int_{\mathbb{R}^{2}}|u|^{p} d x=0 .
$$

By Lemma 2.1, we have the Pohozaev identity for (2.3),

$$
\mu \int_{\mathbb{R}^{2}}|u|^{2} d x-2 \int_{\mathbb{R}^{2}} \frac{h^{2}(|x|)}{|x|^{2}} u^{2} d x+\frac{2 \lambda}{p} \int_{\mathbb{R}^{2}}|u|^{p} d x=0 .
$$


NoDEA

By combining (2.4) and (2.5), we have

$$
\int_{\mathbb{R}^{2}}|\nabla u|^{2} d x+\int_{\mathbb{R}^{2}} \frac{h^{2}(|x|)}{|x|^{2}} u^{2} d x-\lambda \frac{p-2}{p} \int_{\mathbb{R}^{2}}|u|^{p} d x=0,
$$

and

$$
\frac{2}{p}\|\nabla u\|_{L^{2}}^{2}+\left(1-\frac{2}{p}\right) \mu \int_{\mathbb{R}^{2}}|u|^{2} d x+\left(\frac{6}{p}-2\right) \int_{\mathbb{R}^{2}} \frac{h^{2}(|x|)}{|x|^{2}} u^{2} d x=0 .
$$

When $2<p \leq 3$, by (2.7), we have

$$
0 \leq\left(1-\frac{2}{p}\right) \mu \int_{\mathbb{R}^{2}}|u|^{2} d x=-\frac{2}{p}\|\nabla u\|_{L^{2}}^{2}+\left(2-\frac{6}{p}\right) \int_{\mathbb{R}^{2}} \frac{h^{2}(|x|)}{|x|^{2}} u^{2} d x<0,
$$

when $u \neq 0$, contradiction.

By (2.6), we have $0 \geq \int_{\mathbb{R}^{2}}|\nabla u|^{2} d x-\frac{p-2}{p} \lambda\|\nabla u\|_{L^{2}}^{p-2}\|u\|_{L^{2}}^{p(1-\gamma)}$, where $\gamma=$ $2\left(\frac{1}{2}-\frac{1}{p}\right)$. Correspondingly,

$$
\|\nabla u\|_{L^{2}}^{(4-p)} \leq C(p)\|u\|_{L^{2}}^{p(1-\gamma)} .
$$

When $3<p<4$, and $\|u\|_{L^{2}} \leq c$, with $c$ sufficiently small.

If $\mu=0$, then we can use Proposition 4.1 in [5] to get a contradiction if $u \neq 0$ with $J(u)<0$.

If $\mu>0$, then

$$
\begin{aligned}
0 & <\left(1-\frac{2}{p}\right) \mu \int_{\mathbb{R}^{2}}|u|^{2} d x \\
& =-\frac{2}{p}\|\nabla u\|_{L^{2}}^{2}+\left(2-\frac{6}{p}\right) \int_{\mathbb{R}^{2}} \frac{h^{2}(|x|)}{|x|^{2}} u^{2} d x \\
& \leq\left(2-\frac{6}{p}\right) \int_{\mathbb{R}^{2}} \frac{h^{2}(|x|)}{|x|^{2}} u^{2} d x \leq C\|u\|_{L^{2}}^{4} .
\end{aligned}
$$

To derive the last inequality above, we used the boundedness of $\|\nabla u\|_{L^{2}}$, which can be seen from (2.8). We see that when $\|u\|_{L^{2}}$ is sufficiently small, the above inequality (2.9) will lead to a contradiction.

When $p>4$, and $\|u\|_{L^{2}} \leq c$, with $c$ sufficiently small. By Lemma 2.3, we have

$$
\begin{aligned}
& \left(1-\frac{2}{p}\right) \mu \int_{\mathbb{R}^{2}}|u|^{2} d x \\
= & -\frac{2}{p}\|\nabla u\|_{L^{2}}^{2}+\left(2-\frac{6}{p}\right) \int_{\mathbb{R}^{2}} \frac{h^{2}(|x|)}{|x|^{2}} u^{2} d x \\
\leq & -\frac{2}{p}\|\nabla u\|_{L^{2}}^{2}+C\left(2-\frac{6}{p}\right)\|\nabla u\|_{L^{2}}^{2}\|u\|_{L^{2}}^{4} .
\end{aligned}
$$

When $\|u\|_{L^{2}}$ is sufficiently small, the right hand side of (2.10) is negative, while the left hand side of (2.10) is nonnegative, a contradiction. So we complete the proof of the Lemma. 


\section{Solutions with fixed $L^{2}$ norm}

\subsection{The minimax structure}

3.1.1. $2<\boldsymbol{p}<4$. In this case, by Proposition 2.4, (4.6) and (4.7) in [5], we have

$$
J(u) \geq \frac{1}{4} \int_{\mathbb{R}^{2}}|\nabla u|^{2} d x+\frac{1}{4} \int_{\mathbb{R}^{2}} \frac{u^{2}}{|x|^{2}}\left(\int_{0}^{|x|} \frac{s}{2} u^{2}(s)\right)^{2} d x-C \int_{\mathbb{R}^{2}}|u|^{2} d x,
$$

so $J(u)$ is bounded from below on $S(c)$. Also we have the transformation $v \rightarrow v_{t}(x) \equiv t v(t x)$, under which the $L^{2}$ norm is invariant. Moreover

$$
J\left(v_{t}\right)=t^{2}\left(\frac{1}{2} \int_{\mathbb{R}^{2}}|\nabla v|^{2} d x+\frac{1}{2} \int_{\mathbb{R}^{2}} \frac{v^{2}}{|x|^{2}}\left(\int_{0}^{|x|} \frac{s}{2} v^{2}(s)\right)^{2} d x-t^{p-4} \frac{\lambda}{p} \int_{\mathbb{R}^{2}}|u|^{p} d x\right) .
$$

We have, $\forall z \in S(c)$, there exists a unique $T=T(z)>0$ such that

$$
J\left(z_{T(z)}\right)=0, \quad \forall 0<t<T(z), J\left(z_{t}\right)<0, \quad \text { and } \quad \forall t>T(z), J\left(z_{t}\right)>0 .
$$

For any $k \in \mathbb{N}$, we choose $E_{k}$ a $k$-dimensional subspace of $H_{r}^{1}$, such that $E_{k} \subset E_{k^{\prime}}$ for $k<k^{\prime}$ and set $S_{k}=E_{k} \cap S(c)$. In particular, we have $\forall z \in S_{k}$, $\exists T_{z}^{\prime} \equiv \frac{1}{2} T(z)>0$, such that $J\left(z_{T_{z}^{\prime}}\right)<0$ and the map $z \rightarrow T_{z}^{\prime}$ is continuous. We see the map $S_{k} \ni z \rightarrow \tau(z):=z_{T_{z}^{\prime}}$ is odd and continuous. For any closed and symmetric set $A \subset H_{r}^{1} \backslash\{0\}$, let $\gamma(A)$ denotes the Krasnoselski genus of $A$. By the discussion above, we have $\gamma\left(\tau\left(S_{k}\right)\right) \geq k$, and $\tau\left(S_{k}\right) \subset\left\{u \in H_{r}^{1}: J(u)<0\right\}$. Therefore, setting

$$
d_{k}=\inf _{\gamma(A) \geq k, A \subset S(c)} \sup \{J(u): u \in A\}, \quad k \in \mathbb{N} .
$$

One has that $d_{k}<0, k \in \mathbb{N}$. Then the Lusternik-Schnirelmann theory yields $\mathrm{P}-\mathrm{S}$ sequences $\pm v_{k}^{n}$ such that

$$
J\left( \pm v_{k}^{n}\right) \rightarrow d_{k}<0,\left.\quad J\right|_{S(c)} ^{\prime}\left( \pm v_{k}^{n}\right) \rightarrow 0, \quad \text { as } \quad n \rightarrow \infty, \quad k \in \mathbb{N} .
$$

In Sect. 3.2, we will show that these $\mathrm{P}-\mathrm{S}$ sequences converge. It is well-known that $-\infty<d_{1} \leq d_{2} \leq \cdots \leq d_{k} \leq \cdots<0$, and if two of these numbers coincide, then the corresponding critical set is infinite, see e.g. [23]. So there will be infinitely many solutions in this case.

3.1.2. $\boldsymbol{p}>$ 4. Let $\left\{V_{n}\right\} \subset H_{r}^{1}\left(\mathbb{R}^{2}\right)$ be a strictly increasing sequence of finitedimensional linear subspace in $H_{r}^{1}\left(\mathbb{R}^{2}\right)$, such that $\cup_{n} V_{n}$ is dense in $H_{r}^{1}\left(\mathbb{R}^{2}\right)$. We denote by $V_{n}^{\perp}$ the orthogonal space of $V_{n}$ in $H_{r}^{1}\left(\mathbb{R}^{2}\right)$. Then

Lemma 3.1. ([3], Lemma 2.1). Assume that $p \in(4,+\infty)$, then we have

$$
\mu_{n}:=\inf _{u \in V_{n-1}^{\perp}} \frac{\int_{\mathbb{R}^{2}}\left(|\nabla u|^{2}+|u|^{2}\right) d x}{\left(\int_{\mathbb{R}^{2}}|u|^{p} d x\right)^{2 / p}}=\inf _{u \in V_{n-1}^{\perp}} \frac{\|u\|_{H^{1}}^{2}}{\|u\|_{L^{p}\left(\mathbb{R}^{2}\right)}^{2}} \rightarrow \infty, \quad \text { as } \quad n \rightarrow \infty .
$$


Now fix $c>0$ and for each $n \in N^{+}$, we define

$$
\rho_{n}:=L^{-\frac{1}{p-2}} \mu_{n}^{\frac{1}{p-2}}, \quad \text { with } \quad L=\max _{x>0} \frac{\left(x^{2}+c^{2}\right)^{p / 2}}{x^{p}+c^{p}},
$$

and $B_{n}:=\left\{u \in V_{n-1}^{\perp} \cap S(c):\|\nabla u\|_{L^{2}}=\rho_{n}\right\}$. Define also

$$
b_{n}:=\inf _{u \in B_{n}} J(u) \text {. }
$$

Then we have

Lemma 3.2. Let $p \in(4, \infty)$, then $b_{n} \rightarrow+\infty$ as $n \rightarrow \infty$.

Proof. For any $u \in B_{n}$

$$
\begin{aligned}
J(u) & =\frac{1}{2}\|\nabla u\|_{L^{2}}^{2}+\frac{1}{2} \int_{\mathbb{R}^{2}} \frac{u^{2}}{|x|^{2}}\left(\int_{0}^{|x|} \frac{s}{2} u^{2}(s) d s\right)^{2} d x-\frac{\lambda}{p} \int_{\mathbb{R}^{2}}|u|^{p} d x \\
& \geq \frac{1}{2}\|\nabla u\|_{L^{2}}^{2}-\frac{1}{p \mu_{n}}\left(\|\nabla u\|_{L^{2}}^{2}+c^{2}\right)^{\frac{p}{2}} \\
& \geq \frac{1}{2}\|\nabla u\|_{L^{2}}^{2}-\frac{L}{p \mu_{n}}\left(\|\nabla u\|_{L^{2}}^{p}+c^{p}\right) \\
& \geq\left(\frac{1}{2}-\frac{1}{p}\right) \rho_{n}^{2}-\frac{L}{p \mu_{n}} c^{p} .
\end{aligned}
$$

Since $p>2$, so by Lemma 3.1, we have $b_{n} \rightarrow+\infty$ as $n \rightarrow+\infty$.

In the following, we assume that $H \equiv L^{2}\left(\mathbb{R}^{2}\right)$ and $E \equiv H^{1}\left(\mathbb{R}^{2}\right)$ with their scalar products denoted by $\langle\cdot, \cdot\rangle_{H}$ and $\langle\cdot, \cdot\rangle_{E}$, the corresponding norms are $\|\cdot\|_{H}$ and $\|\cdot\|_{E}$. Also we let $E \times \mathbb{R}$ be equipped with the scalar product $\langle\cdot, \cdot\rangle_{E \times \mathbb{R}}=\langle\cdot, \cdot\rangle_{E}+\langle\cdot, \cdot\rangle_{\mathbb{R}}$, and corresponding norm $\|\cdot\|_{E \times \mathbb{R}}=\left\{\|\cdot\|_{E}^{2}+|\cdot|_{\mathbb{R}}^{2}\right\}^{\frac{1}{2}}$, and $\mathbf{E}=E \times \mathbb{R},\|\cdot\|_{\mathbf{E}}=\|\cdot\|_{E \times \mathbb{R}}$ for short. We let $H: E \times \mathbb{R} \rightarrow E$, $H(u, m)(x)=e^{m} u\left(e^{m} x\right)$.

Lemma 3.3. Let $u \in S(c)$ be arbitrary but fixed, then we have

(1) $\|\nabla H(u, m)\|_{L^{2}} \rightarrow 0$ and $J(H(u, m)) \rightarrow 0$ as $m \rightarrow-\infty$.

(2) $\|\nabla H(u, m)\|_{L^{2}} \rightarrow+\infty$ and $J(H(u, m)) \rightarrow-\infty$ as $m \rightarrow+\infty$.

Proof. We have

$$
\|H(u, m)\|_{L^{2}}=c \quad \text { and } \quad\|\nabla H(u, m)\|_{L^{2}}=e^{m}\|\nabla u\|_{L^{2}} .
$$

Now we have

$$
\begin{aligned}
& J(H(u, m)) \\
& =\frac{1}{2}\|\nabla H(u, m)\|_{L^{2}}^{2}+\frac{1}{2} \int_{\mathbb{R}^{2}} \frac{H(u, m)^{2}}{|x|^{2}}\left(\int_{0}^{|x|} \frac{s}{2} H(u, m)^{2} d s\right)^{2} d x \\
& -\frac{\lambda}{p} \int_{\mathbb{R}^{2}}|H(u, m)|^{p} d x \\
& =\frac{1}{2} e^{2 m}\|\nabla u\|_{L^{2}}^{2}+\frac{1}{2} e^{2 m} \int_{\mathbb{R}^{2}} \frac{u^{2}}{|x|^{2}}\left(\int_{0}^{|x|} \frac{s}{2} u^{2} d s\right)^{2} d x-\frac{\lambda}{p} e^{(p-2) m} \int_{\mathbb{R}^{2}}|u|^{p} d x .
\end{aligned}
$$


We see, as $m \rightarrow-\infty,\|\nabla H(u, m)\|_{L^{2}} \rightarrow 0$ and $J(H(u, m)) \rightarrow 0$. Since $p-2>2$, so as $m \rightarrow+\infty,\|\nabla H(u, m)\|_{L^{2}} \rightarrow+\infty$ and $J(H(u, m)) \rightarrow-\infty$.

Lemma 3.4. There exists $\rho_{0}, \rho_{0}^{\prime}$, such that when $0<\rho_{0}<\rho_{0}^{\prime}<\rho_{n}$,

$$
0<\sup _{u \in A} J(u)<\inf _{u \in B} J(u)=: b_{0},
$$

with $A=\left\{u \in S(c),\|\nabla u\|_{L^{2}}^{2} \leq \rho_{0}\right\}, B=\left\{u \in S(c),\|\nabla u\|_{L^{2}}^{2}=\rho_{0}^{\prime}\right\}$.

Proof. By Lemma 2.3, we have

$$
J(u) \leq \frac{1}{2}\|\nabla u\|_{L^{2}}^{2}+C\|\nabla u\|_{L^{2}}^{2}\|u\|_{L^{2}}^{4} .
$$

We see when $\|\nabla u\|_{L^{2}} \rightarrow 0, J(u) \rightarrow 0$. On the other hand, by the GagliardoNirenberg inequality, we have

$$
\begin{aligned}
J(u) & \geq \frac{1}{2}\|\nabla u\|_{L^{2}}^{2}-C\|\nabla u\|_{L^{2}}^{p \gamma}\|u\|_{L^{2}}^{p(1-\gamma)} \\
& \geq C\|\nabla u\|_{L^{2}}^{2}\left(1-\|\nabla u\|_{L^{2}}^{p \gamma-2}\|u\|_{L^{2}}^{p(1-\gamma)}\right),
\end{aligned}
$$

with $\gamma=2\left(\frac{1}{2}-\frac{1}{p}\right)$. We see that when $\|\nabla u\|_{L^{2}}$ is sufficiently small, $J(u) \geq$ $C\|\nabla u\|_{L^{2}}^{2}$. By combining these two facts, we prove the Lemma.

By Lemma 3.3, there exists $m_{n}>0$ such that

$$
\bar{\gamma}_{n}:[0,1] \times\left(S \cap V_{n}\right) \rightarrow S, \bar{\gamma}_{n}(t, u)=H\left(u, 2 m_{n} t-m_{n}\right)
$$

satisfies:

$$
\begin{aligned}
& \left|\nabla\left(\bar{\gamma}_{n}(0, u)\right)\right|_{2}<\rho_{0}<\rho_{n},\left|\nabla\left(\bar{\gamma}_{n}(1, u)\right)\right|_{2}>\rho_{n}, \quad \text { and } \\
& 0<J\left(\bar{\gamma}_{n}(0, u)\right)<\max \left\{b_{0}, b_{n}\right\}, J\left(\bar{\gamma}_{n}(1, u)\right)<b_{n},
\end{aligned}
$$

uniformly for $u \in S \cap V_{n}$. Now we define

$$
\begin{aligned}
\Gamma_{n}= & \left\{\gamma:[0,1] \times\left(S \cap V_{n}\right) \rightarrow S \mid \gamma \text { is continuous, odd in } u\right. \text { and such that } \\
& \left.\forall u: \gamma(0, u)=\bar{\gamma}_{n}(0, u), \gamma(1, u)=\bar{\gamma}_{n}(1, u)\right\}
\end{aligned}
$$

Clearly, we have $\tilde{\gamma}_{n} \in \Gamma_{n}$. And in [3], the authors prove the following Lemma:

Lemma 3.5. For every $\gamma \in \Gamma_{n}$, there exists $(t, u) \in[0,1] \times\left(S \cap V_{n}\right)$ such that $\gamma(t, u) \in B_{n}$.

By Lemma 3.5, we have

$$
c_{n}=\inf _{\gamma \in \Gamma_{n}} \max _{t \in[0,1], u \in S \cap V_{n}} J(\gamma(t, u)) \geq b_{n}=\inf _{u \in B_{n}} J(u) \rightarrow \infty .
$$

Clearly, by Lemmas 3.3 and 3.4 , we have $c_{n} \geq b_{0}>0$. Now we shall prove that $\left\{c_{n}\right\}$ is a set of critical values of $J$ restricted to $S(c)$.

We fix $n$ from now on.

Proposition 3.6. Given $c>0$, there exists a Palais-Smale sequence $\left\{v_{k}\right\} \subset$ $S(c)$ satisfying $J\left(v_{k}\right) \rightarrow c_{n},\left.J^{\prime}\right|_{S(c)}\left(v_{k}\right) \rightarrow 0$, as $k \rightarrow \infty$, and

$$
\left\|\nabla v_{k}\right\|_{L^{2}}^{2}+\int_{\mathbb{R}^{2}} \frac{\left|v_{k}\right|^{2}}{|x|^{2}}\left(\int_{0}^{|x|} \frac{s}{2} v_{k}^{2} d s\right)^{2} d x-\frac{\lambda(p-2)}{p} \int_{\mathbb{R}^{2}}\left|v_{k}\right|^{p} d x \rightarrow 0 .
$$


To prove Proposition 3.6, following [14], we introduce the auxiliary functional

$$
\tilde{J}: S(c) \times \mathbb{R} \rightarrow \mathbb{R},(u, m) \rightarrow J(H(u, m)),
$$

and define

$\tilde{\Gamma}_{n}:=\left\{\tilde{\gamma}:[0,1] \times\left(S \cap V_{n}\right) \rightarrow \mathbb{R} \times S \mid \tilde{\gamma}\right.$ is continuous, odd in $u$, and such that $\left.H \circ \tilde{\gamma} \in \Gamma_{n}\right\}$.

Clearly, for any $\gamma \in \Gamma_{n}, \tilde{\gamma}: H(\gamma, 0) \in \tilde{\Gamma}_{n}$. Define

$$
\tilde{c}_{n}:=\inf _{\tilde{\gamma} \in \tilde{\Gamma}_{n}} \max _{t \in[0,1], u \in S(c) \cap V_{n}} \tilde{J}(\tilde{\gamma}(t, u)) .
$$

Then we have $\tilde{c}_{n}=c_{n}$. Indeed, this can from the fact that there are maps

$$
\Phi: \Gamma_{n} \rightarrow \tilde{\Gamma}_{n}, \gamma \rightarrow[(0, \gamma):(t, u) \rightarrow(0, \gamma(t, u))]
$$

and

$$
\Psi: \tilde{\Gamma}_{n} \rightarrow \Gamma_{n}, \tilde{\gamma} \rightarrow[H \circ \tilde{\gamma}:(t, u) \rightarrow H \circ(\tilde{\gamma}(t, u))]
$$

satisfy

$$
\tilde{J}(\Phi(\gamma)(t, u))=J(\gamma(t, u)) \text { and } J(\Psi(\tilde{\gamma})(t, u))=\tilde{J}(\tilde{\gamma}(t, u)) .
$$

In [14], by using the Ekeland's variational principle, the author establish the following Lemma.

Lemma 3.7. Let $\epsilon>0$, and $\tilde{\gamma}_{0} \in \tilde{\Gamma}_{n}$ be such that

$$
\max _{t \in[0,1], u \in S(c) \cap V_{n}} \tilde{J}\left(\tilde{\gamma}_{0}(t, u)\right) \leq \tilde{c}_{n}+\epsilon
$$

then there exists a pair of $\left(u_{0}, m_{0}\right) \in S(c) \times \mathbb{R}$ such that:

(1) $\tilde{J}\left(u_{0}, m_{0}\right) \in\left[\tilde{c}_{n}-\epsilon, \tilde{c}_{n}+\epsilon\right]$.

(2) $\min _{t \in[0,1], u \in S(c) \cap V_{n}}\left\|\left(u_{0}, m_{0}\right)-\tilde{\gamma}_{0}(t, u)\right\|_{E} \leq \sqrt{\epsilon}$.

(3) $\left\|\left.\tilde{J}^{\prime}\right|_{S(c) \times R}\left(u_{0}, m_{0}\right)\right\|_{\mathbf{E}^{*} \times \mathbf{E}} \leq 2 \sqrt{\epsilon}$, i.e.

$$
\left|\left\langle\tilde{J}^{\prime}\left(u_{0}, m_{0}\right), z\right\rangle_{\mathbf{E}^{*} \times \mathbf{E}}\right| \leq 2 \sqrt{\epsilon}\|z\|_{E},
$$

holds for all $z \in \tilde{T}_{\left(u_{0}, m_{0}\right)}:=\left\{\left(z_{1}, z_{2}\right) \in \mathbf{E},\left\langle u_{0}, z_{1}\right\rangle_{L^{2}}=0\right\}$.

Proof of the Proposition 3.6. From the definition of $c_{n}$, we have, for each $k \in$ $\mathbb{N}$, there exists a $\gamma_{k} \in \Gamma_{n}$ such that

$$
\max _{t \in[0,1], u \in S(c) \cap V_{n}} J\left(\gamma_{k}(t, u)\right) \leq c_{n}+\frac{1}{k} .
$$

Since $\tilde{c}_{n}=c_{n}, \tilde{\gamma}_{k}=\left(\gamma_{k}, 0\right) \in \tilde{\Gamma}_{n}$ satisfies that

$$
\max _{t \in[0,1], u \in S(c) \cap V_{n}} \tilde{J}\left(\tilde{\gamma}_{k}(t, u)\right) \leq \tilde{c}_{n}+\frac{1}{k} .
$$


By Lemma 3.7, we know there exists a sequence $\left\{u_{k}, m_{k}\right\} \subset S(c) \times \mathbb{R}$ such that

$$
\begin{aligned}
& \text { (1) } \tilde{J}\left(u_{k}, m_{k}\right) \in\left[c_{n}-\frac{1}{k}, c_{n}+\frac{1}{k}\right] . \\
& \text { (2) } \min _{t \in[0,1], u \in S(c) \cap V_{n}}\left\|\left(u_{k}, m_{k}\right)-\left(\gamma_{k}(t, u), 0\right)\right\|_{\mathbf{E}} \leq \frac{1}{\sqrt{k}} . \\
& \text { (3) }\left\|\left.\tilde{J}^{\prime}\right|_{S(c) \times R}\left(u_{k}, m_{k}\right)\right\|_{\mathbf{E}^{*}} \leq \frac{2}{\sqrt{k}} \text {, i.e. } \\
& \left|\left\langle\tilde{J}^{\prime}\left(u_{k}, m_{k}\right), z\right\rangle_{\mathbf{E}^{*} \times \mathbf{E}}\right| \leq \frac{2}{\sqrt{k}}\|z\|_{E},
\end{aligned}
$$

holds for all $z \in \tilde{T}_{\left(u_{k}, m_{k}\right)}:=\left\{\left(z_{1}, z_{2}\right) \in \mathbf{E},\left\langle u_{k}, z_{1}\right\rangle_{L^{2}}=0\right\}$.

Now we let $v_{k}=H\left(u_{k}, m_{k}\right)$. Since $J\left(v_{k}\right)=J\left(H\left(u_{k}, m_{k}\right)\right)=\tilde{J}\left(u_{k}, m_{k}\right)$, so we have $J\left(v_{k}\right) \rightarrow c_{n}$, as $k \rightarrow \infty$, and we prove (1).

Let $\partial_{m} \tilde{J}\left(u_{k}, m_{k}\right) \equiv\left\langle\tilde{J}^{\prime}\left(u_{k}, m_{k}\right),(0,1)\right\rangle_{\mathbf{E}^{*} \times \mathbf{E}}$, then we have $\partial_{m} \tilde{J}\left(u_{k}, m_{k}\right)$ $\rightarrow 0$ as $k \rightarrow \infty$.

By calculating, we have

$$
\begin{aligned}
& \partial_{m} \tilde{J}\left(u_{k}, m_{k}\right) \\
&=e^{2 m_{k}} \int_{\mathbb{R}^{2}}\left|\nabla u_{k}\right|^{2} d x+e^{2 m_{k}} \int_{\mathbb{R}^{2}} \frac{u_{k}^{2}}{|x|^{2}}\left(\int_{0}^{|x|} \frac{s}{2} u_{k}^{2} d s\right)^{2} d x \\
&-\frac{\lambda}{p} e^{(p-2) m_{k}}(p-2) \int_{\mathbb{R}^{2}}\left|u_{k}\right|^{p} d x \\
&= \int_{\mathbb{R}^{2}}\left|\nabla v_{k}\right|^{2} d x+\int_{\mathbb{R}^{2}} \frac{v_{k}^{2}}{|x|^{2}}\left(\int_{0}^{|x|} \frac{s}{2} v_{k}^{2} d s\right)^{2} d x-\lambda \frac{p-2}{p} \int_{\mathbb{R}^{2}}\left|v_{k}\right|^{p} d x,
\end{aligned}
$$

and we get (3.7).

Now let $w \in T_{v_{k}}$, we have

$$
\begin{aligned}
\left\langle J^{\prime}\left(v_{k}\right), w\right\rangle_{E^{*} \times E} & \int_{\mathbb{R}^{2}} \nabla v_{k} \nabla w(x) d x+\int_{\mathbb{R}^{2}}\left(\int_{|x|}^{\infty} \frac{h_{1}(s)}{s} v_{k}^{2} d s v_{k}+\frac{h_{1}^{2}(|x|)}{|x|^{2}} v_{k}\right) w d x \\
& -\lambda \int_{\mathbb{R}^{2}}\left|v_{k}\right|^{p-2} v_{k} w d x \\
= & e^{2 m_{k}} \int_{\mathbb{R}^{2}} \nabla u_{k}\left(e^{m_{k}} x\right) \nabla w(x) d x+e^{5 m_{k}} \\
& \times \int_{\mathbb{R}^{2}}\left(\int_{|x|}^{\infty} \frac{h_{2}(s)}{s} u_{k}^{2}\left(e^{m_{k}} x\right) d s u_{k}\left(e^{m_{k}} x\right)\right. \\
& \left.+\frac{h_{2}^{2}(|x|)}{|x|^{2}} u_{k}\left(e^{m_{k}} x\right)\right) w d x-\lambda e^{(p-1) m_{k}} \int_{\mathbb{R}^{2}}\left|u_{k}\left(e^{m_{k}} x\right)\right|^{p-2} u_{k}\left(e^{m_{k}} x\right) w d x \\
= & e^{m_{k}} \int_{\mathbb{R}^{2}} \nabla u_{k}(x) \nabla w\left(e^{-m_{k}} x\right) d x
\end{aligned}
$$




$$
\begin{aligned}
& +e^{m_{k}} \int_{\mathbb{R}^{2}}\left(\int_{|x|}^{\infty} \frac{h_{3}(s)}{s} u_{k}^{2} d s u_{k}+\frac{h_{3}^{2}(|x|)}{|x|^{2}} u_{k}\right) w\left(e^{-m_{k}} x\right) d x \\
& -\lambda e^{(p-3) m_{k}} \int_{\mathbb{R}^{2}}\left|u_{k}\right|^{p-2} u_{k} w\left(e^{-m_{k}} x\right) d x,
\end{aligned}
$$

where in the above, $h_{1}(s)=\int_{0}^{s} \frac{t}{2} v_{k}^{2}(t) d t, h_{2}(s)=\int_{0}^{s} \frac{t}{2} u_{k}^{2}\left(e^{m_{k}} t\right) d t, h_{3}(s)=$ $\int_{0}^{s} \frac{t}{2} u_{k}^{2}(t) d t$. Thus setting $\tilde{w}(x) \equiv e^{-m_{k}} w\left(e^{-m_{k}} x\right)$, we have

$$
\left\langle J^{\prime}\left(v_{k}\right), w\right\rangle_{E^{*} \times E}=\left\langle\tilde{J}^{\prime}\left(u_{k}, m_{k}\right),(\tilde{w}, 0)\right\rangle_{\mathbf{E}^{*} \times \mathbf{E}} .
$$

Since

$$
\begin{aligned}
& (\tilde{w}, 0) \in \tilde{T}\left(u_{k}, m_{k}\right) \\
\Leftrightarrow & \left\langle\tilde{w}, u_{k}\right\rangle_{L^{2}}=0 \Leftrightarrow \int_{\mathbb{R}^{2}} u_{k}(x) e^{-m_{k}} w\left(e^{-m_{k}} x\right) d x=0 \\
\Leftrightarrow & \int_{\mathbb{R}^{2}} e^{m_{k}} u_{k}\left(e^{m_{k}} x\right) w(x) d x=0 \\
\Leftrightarrow & \left\langle v_{k}, w\right\rangle_{L^{2}}=0 \Leftrightarrow w \in T_{v_{k}},
\end{aligned}
$$

so we have $(\tilde{w}, 0) \in \tilde{T}\left(u_{k}, m_{k}\right)$. By (3.11) and (3.12), we have $\mid\left\langle J^{\prime}\left(v_{k}\right)\right.$, $w\rangle_{E^{*} \times E} \mid \leq \frac{2}{\sqrt{k}}\|(\tilde{w}, 0)\|_{\mathbf{E}}$. In the following we will show that for $k$ sufficiently large, $\|(\tilde{w}, 0)\|_{\mathbf{E}} \leq 2\|w\|_{E}$, then we conclude Proposition 3.6. Indeed we have

$$
\left|m_{k}\right|=\left|m_{k}-0\right| \leq \min _{t \in[0,1], u \in S(c) \cap V_{n}}\left\|\left(v_{k}, m_{k}\right)-\left(\gamma_{k}(t, u), 0\right)\right\|_{\mathbf{E}} \leq \frac{1}{\sqrt{k}} .
$$

So for $k$ sufficiently large,

$$
\begin{aligned}
& \|(\tilde{w}, 0)\|_{\mathbf{E}}^{2} \\
& \quad=\|\tilde{w}\|_{H^{1}}^{2}=\int_{\mathbb{R}^{2}}|\tilde{w}(x)|^{2} d x+\int_{\mathbb{R}^{2}}|\nabla \tilde{w}(x)|^{2} d x \\
& \quad=\int_{\mathbb{R}^{2}}|\tilde{w}(x)|^{2} d x+e^{-2 m_{k}} \int_{\mathbb{R}^{2}}|\nabla w(x)|^{2} d x \\
& \quad \leq 2\|w\|_{E}^{2} .
\end{aligned}
$$

\subsection{Compactness}

Since the boundedness of the $\mathrm{P}-\mathrm{S}$ sequences is easy for $2<p<4$, and to get the conclusions in the following Lemma 3.8 for the case $2<p<4$ is similar to the $p>4$ case, we will mainly discuss in the case $p>4$.

By (3.7), we have

$$
\left\|\nabla v_{k}\right\|_{L^{2}}^{2}+\int_{\mathbb{R}^{2}} \frac{\left|v_{k}\right|^{2}}{|x|^{2}}\left(\int_{0}^{|x|} \frac{s}{2} v_{k}^{2} d s\right)^{2} d x-\frac{\lambda(p-2)}{p} \int_{\mathbb{R}^{2}}\left|v_{k}\right|^{p} d x \rightarrow 0 .
$$

Also

$$
\tilde{J}\left(v_{k}\right)=\frac{1}{2}\left\|\nabla v_{k}\right\|_{L^{2}}^{2}+\frac{1}{2} \int_{\mathbb{R}^{2}} \frac{\left|v_{k}\right|^{2}}{|x|^{2}}\left(\int_{0}^{|x|} \frac{s}{2} v_{k}^{2} d s\right)^{2} d x-\frac{\lambda}{p} \int_{\mathbb{R}^{2}}\left|v_{k}\right|^{p} d x \rightarrow c_{n} .
$$


Since

$$
\int_{\mathbb{R}^{2}}\left|v_{k}\right|^{p} d x=\frac{2 p}{\lambda(p-4)}\left((3.14)-\frac{1}{2} \times(3.13)\right),
$$

so there exists constants $c_{1}$ and $c_{2}$, such that

$$
0<c_{1} \leq \int_{\mathbb{R}^{2}}\left|v_{k}\right|^{p} d x \leq c_{2}
$$

By using (3.13) again, $\frac{1}{2}\left\|\nabla v_{k}\right\|_{L^{2}}^{2}+\int_{\mathbb{R}^{2}} \frac{\left|v_{k}\right|^{2}}{|x|^{2}}\left(\int_{0}^{|x|} \frac{s}{2} v_{k}^{2} d s\right)^{2} d x$ is also bounded consequently. So up to a subsequence, there exists $v_{n} \in E$, such that $v_{k} \rightarrow v_{n}$ weakly in $E$ as $k \rightarrow \infty$. By the compact embedding $H_{r}^{1}\left(\mathbb{R}^{2}\right) \hookrightarrow L^{p}\left(\mathbb{R}^{2}\right)$ for $2<p<\infty$ ([24]) and (3.15), we know that $v_{n}$ is nontrivial. Now note that by Proposition 3.6, there exists $C>0$ independent of $k \in N$ such that

$$
\left|\left\langle J^{\prime}\left(v_{k}\right)-\frac{1}{\left\|v_{k}\right\|_{L^{2}}^{2}}\left\langle J^{\prime}\left(v_{k}\right), v_{k}\right\rangle v_{k}, z\right\rangle_{E^{*} \times E}\right| \leq \frac{C}{\sqrt{k}}\|z\|_{E} \quad \text { for all } \quad z \in E,
$$

we choose a subsequence of $\left\{v_{k}\right\}$ in Proposition 3.6 such that $\left\|\left.J^{\prime}\right|_{S(c)}\left(v_{k}\right)\right\|_{E^{*}} \leq$ $\frac{C}{\sqrt{k}}$. Indeed for all $z \in E$,

$$
\left\langle J^{\prime}\left(v_{k}\right)-\frac{1}{\left\|v_{k}\right\|_{L^{2}}^{2}}\left\langle J^{\prime}\left(v_{k}\right), v_{k}\right\rangle v_{k}, z\right\rangle_{E^{*} \times E}=\left\langle J^{\prime}\left(v_{k}\right), P_{v_{k}} z\right\rangle_{E^{*} \times E},
$$

where $P_{v_{k}}$ denotes the orthogonal projector defined in $E$ on the tangent space $T_{v_{k}}$. Since

$$
\begin{aligned}
& \left\|P_{v_{k}} z\right\|_{E} \\
& \quad=\left\|z-\frac{1}{\left\|v_{k}\right\|_{L^{2}}^{2}}\left\langle z, v_{k}\right\rangle v_{k}\right\|_{E} \\
& \quad \leq\|z\|_{E}+\|z\|_{H}\left\|v_{k}\right\| H\left\|v_{k}\right\|_{E} /\left\|v_{k}\right\|_{L^{2}}^{2} \\
& \quad \leq\|z\|_{E}\left(1+\left\|v_{k}\right\|_{E} /\left\|v_{k}\right\|_{L^{2}}\right) .
\end{aligned}
$$

So, $\left\langle J^{\prime}\left(v_{k}\right), P_{v_{k}} z\right\rangle_{E^{*} \times E} \leq \frac{C}{\sqrt{k}}\left\|P_{v_{k}} z\right\|_{E} \leq \frac{C}{\sqrt{k}}\|z\|_{E}$.

Now

$$
\begin{aligned}
& \left\langle J^{\prime}\left(v_{k}\right)-\frac{1}{\left\|v_{k}\right\|_{L^{2}}^{2}}\left\langle J^{\prime}\left(v_{k}\right), v_{k}\right\rangle v_{k}, z\right\rangle_{E^{*} \times E} \\
& =\int_{\mathbb{R}^{2}} \nabla v_{k}(x) \nabla z(x) d x+\int_{\mathbb{R}^{2}}\left(\int_{|x|}^{\infty} \frac{h(s)}{s} v_{k}^{2} d s v_{k}+\frac{h^{2}(|x|)}{|x|^{2}} v_{k}\right) z d x \\
& \quad-\lambda \int_{\mathbb{R}^{2}}\left|v_{k}\right|^{p-2} v_{k} z d x-\lambda_{k} \int_{\mathbb{R}^{2}} v_{k}(x) z(x) d x
\end{aligned}
$$


with

$$
\begin{aligned}
\lambda_{k}= & \frac{1}{\left\|v_{k}\right\|_{L^{2}}^{2}}\left\{\left\|\nabla v_{k}\right\|_{L^{2}}^{2}+\int_{\mathbb{R}^{2}}\left(\int_{|x|}^{\infty} \frac{h(s)}{s} v_{k}^{2}(s) d s\right) v_{k}^{2}\right. \\
& \left.+\frac{h^{2}(|x|)}{|x|^{2}} v_{k}^{2} d x-\lambda \int_{\mathbb{R}^{2}}\left|v_{k}\right|^{p} d x\right\} \\
= & \frac{1}{\left\|v_{k}\right\|_{L^{2}}^{2}}\left\{\left\|\nabla v_{k}\right\|_{L^{2}}^{2}+\int_{\mathbb{R}^{2}} \frac{h^{2}(|x|)}{|x|^{2}} v_{k}^{2} d x-\lambda \int_{\mathbb{R}^{2}}\left|v_{k}\right|^{p} d x\right\},
\end{aligned}
$$

which is bounded. So up to a subsequence $\lambda_{k} \rightarrow \omega_{n}$. By the weak convergence of $\left\{v_{k}\right\}$ and Lemma 2.2, we get the following Lemma:

Lemma 3.8. Assume that $p>2$ and $p \neq 4$, let $\left\{v_{k}\right\} \subset S(c)$ be obtained in Proposition 3.6 and in Sect. 3.1.1. Then there exists $\omega_{n} \in \mathbb{R}$ and $v_{n} \in H_{r}^{1}\left(\mathbb{R}^{2}\right)$, such that, up to a subsequence

(1) $v_{k} \rightarrow v_{n} \neq 0$ in $H_{r}^{1}\left(\mathbb{R}^{2}\right)$,

(2) $-\Delta v_{k}+\omega_{n} v_{k}+\left(\int_{|x|}^{\infty} \frac{h(s)}{s} v_{k}^{2}(s) d s\right) v_{k}+\frac{h^{2}(|x|)}{|x|^{2}} v_{k}-\lambda\left|v_{k}\right|^{p-2} v_{k} \rightarrow$ 0 in $H_{r}^{-1}\left(\mathbb{R}^{2}\right)$,

(3) $-\Delta v_{n}+\omega_{n} v_{n}+\left(\int_{|x|}^{\infty} \frac{h(s)}{s} v_{n}^{2}(s) d s\right) v_{n}+\frac{h^{2}(|x|)}{|x|^{2}} v_{n}-\lambda\left|v_{n}\right|^{p-2} v_{n}=0$ in $H_{r}^{-1}\left(\mathbb{R}^{2}\right)$.

Now, by the principle of symmetric criticality(see e.g. section 1.6 in [25]) and (3) of Lemma 3.8, $v_{n}$ is a weak solution of

$$
-\Delta u+\left(\int_{|x|}^{\infty} \frac{h(s)}{s} u^{2}(s) d s\right) u+\frac{h^{2}(|x|)}{|x|^{2}} u-\lambda|u|^{p-2} u=-\omega_{n} u \quad \text { in } \quad \mathbb{R}^{2} .
$$

If $-\omega_{n} \geq 0$, i.e. $\omega_{n} \leq 0$, by our assumptions on $c$ as in Theorem 1.1, we can use Lemma 2 and deduce $v_{n} \equiv 0$, which contradicts to (1) of Lemma 3.8, so $\omega_{n}>0$.

Now, since $\omega_{n}>0$, by (2) of Lemma 3.8,

$-\Delta v_{k}+\omega_{n} v_{k} \rightarrow-\left(\int_{|x|}^{\infty} \frac{h(s)}{s} v_{k}^{2} d s\right) v_{k}-\frac{h^{2}(|x|)}{|x|^{2}} v_{k}+\lambda\left|v_{k}\right|^{p-2} v_{k}, \quad$ in $H_{r}^{-1}\left(\mathbb{R}^{2}\right)$,

$v_{k} \rightarrow\left(-\Delta+\omega_{n}\right)^{-1}\left(-\left(\int_{|x|}^{\infty} \frac{h(s)}{s} v_{k}^{2} d s\right) v_{k}-\frac{h^{2}(|x|)}{|x|^{2}} v_{k}+\lambda\left|v_{k}\right|^{p-2} v_{k}\right)$ in E.

By the uniqueness of the limit, we have $v_{k} \rightarrow v_{n}$ in $H_{r}^{1}\left(\mathbb{R}^{2}\right)$, in particular $\left\|v_{n}\right\|_{L^{2}\left(\mathbb{R}^{2}\right)}=c$, thus $v_{n} \in S(c)$, and by combining with (3.16), we complete the proof of Theorem 1.1. 


\section{Acknowledgements}

The author would like to deeply thank the anonymous referees for many valuable comments and corrections which improve this paper greatly.

\section{References}

[1] Ambrosetti, A.: On Schrödinger-Poisson systems. Milan J. Math. 76, 257$274(2008)$

[2] Ambrosetti, A., Ruiz, D.: Multiple bound states for the Schrödinger-Poisson problem. Commun. Contemp. Math. 10(3), 391-404 (2008)

[3] Bartsch, T., De Valeriola, S.: Normalized solutions of nonlinear Schrödinger equations. Arch. Math. 100, 75-83 (2013)

[4] Bergé, L., de Bouard, A., Saut, J.C.: Blowing up time-dependent solutions of the planar Chern-Simons gauged nonlinear Schrödinger equation. Nonlinearity 8(2), 235-253(19) 1995

[5] Byeon, J., Huh, H., Seok, J.: Standing waves of nonlinear Schrödinger equations with the gauge field. J. Funct. Anal. 263(6), 1575-1608 (2012)

[6] Bellazzini, J., Jeanjean, L., Luo, T.-J.: Existence and instability of standing waves with prescribed norm for a class of Schrödinger-Poisson equations. Proc. Lond. Math. Soc. (3) 107, 303-339 (2013)

[7] Bellazzini, J., Siciliano, G.: Stable standing waves for a class of nonlinear Schrödinger-Poisson equations. Zeitschrift für Angewandte Mathematik Und Physik. 62(2), 267-280 (2011a)

[8] Bellazzini, J., Siciliano, G.: Scaling properties of functionals and existence of constrained minimizers. J. Funct. Anal. 261(9), 2486-2507 (2011b)

[9] Dunne, G.V.: Self-dual Chern-Simons theories. In: Lecture Notes in Physics Monographs, vol. 36. Springer, Berlin (1995)

[10] Huh, H.: Blow-up solutions of the Chern-Simons-Schrödinger equations. Nonlinearity 22, 967-974 (2009)

[11] Huh, H.: Standing waves of the Schröinger equation coupled with the ChernSimons gauge field. J. Math. Phys. 53, 063702 (2012). doi:10.1063/1.4726192

[12] Huh, H.: Energy solution to the Chern-Simons-Schrödinger equations. In: Crasta, G. (ed.) Abstract and Applied Analysis, vol. 2013, p. 7. Article ID 590653 (2013)

[13] Huang, Y., Liu, Z., Wu, Y.: Existence of prescribed $L^{2}$-norm solutions for a class of Schrödinger-Poisson equation, vol. 2013, p. 11. Article ID 398164. In: Abstract and Applied Analysis. doi:10.1155/2013/398164

[14] Jeanjean, L.: Existence of solutions with prescribed norm for semilinear elliptic equations. Nonlinear Anal. Theory Methods Appl. 28(10), 1633-1659 (1997) 
[15] Jackiw, R., Pi, S.-Y.: Classical and quantal nonrelativistic Chern-Simons theory. Phys. Rev. D. 42, 3500-3513 (1990)

[16] Jackiw, R., Pi, S.-Y.: Self-dual Chern-Simons solitons. Prog. Theor. Phys. Suppl. 107, 1-40 (1992)

[17] Jeanjean, L., Luo, T.: Sharp nonexistence results of prescribed $L^{2}$-norm solutions for some class of Schrödinger-Poisson and quasi-linear equations. Zeitschrift für Angewandte Mathematik Und Physik. 64(4), 937-954 (2013)

[18] Lions, P.-L.: The concentration-compactness principle in the calculus of variation. The locally compact case, part I. Annales de l'Institut Henri Poincare. 1(2), 109-145 (1984a)

[19] Lions, P.-L.: The concentration-compactness principle in the calculus of variation. The locally compact case, part II. Annales de l'Institut Henri Poincare. 1(4), 223-283 (1984b)

[20] Luo, T.-J.: Multiplicity of normalized solutions for a class of nonlinear Schrödinger-Poisson-Slater equations. J. Math. Anal. Appl. 416(1), 195204 (2014). doi:10.1016/j.jmaa.2014.02.038

[21] Pomponio, A., Ruiz, D.: A variational analysis of a gauged nonlinear Schrödinger equation. Preprint. arXiv:1306.2051

[22] Ruiz, D.: The Schrödinger-Poisson equation under the effect of a nonlinear local term. J. Funct. Anal. 237(2), 655-674 (2006)

[23] Struwe, M: Variational Methods. Applications to Nonlinear Partial Differential Equations and Hamiltonian Systems, 3rd edn, 34. Springer-Verlag, Berlin (1996)

[24] Strauss, W.-A.: Existence of solitary waves in higher dimensions. Commun. Math. Phys. 55, 149-162 (1977)

[25] Willem, M.: Minimax Methods. Birkhäuser, Boston (1996)

[26] Zhao, L., Zhao, F.: Positive solutions for Schrödinger-Poisson equations with a critical exponent. Nonlinear Anal. Theory Methods Appl. 70(6), 21502164 (2009). doi:10.1016/j.na.2008.02.116

Jianjun Yuan

The College of Information and Technology

Nanjing University of Chinese Medicine

Nanjing 210046

China

e-mail: yuanjjn2@163.com

Received: 10 March 2015.

Accepted: 19 July 2015. 